\title{
A Study on the Icon Feedback Types of Small Touch Screen for the Elderly
}

\author{
Wang-Chin Tsai and Chang-Franw Lee \\ Graduate School of Design, National Yunlin University of Science and Technology \\ 123, University Road Section 3, Touliu, Yunlin, 64002, Taiwan, R.O.C. \\ \{g9330802, leecf $\}$ @yuntech. edu.tw
}

\begin{abstract}
Small touch screens are widely used in applications such as bank ATMs, point-of-sale terminals, ticket vending machines, facsimiles, and home automation in the daily life. It is intuition-oriented and easy to operate. There are a lot of elements that affect the small screen touch performance. One of the essential parts is icon feedback. However, to merely achieve beautiful icon feedback appearance and create interesting interaction experience, many interface designers ignore the real user needs. It is critical for them to trade off the icon feedback type associated with the different users needs in the touch interaction. This is especially important when the user capability is very limited. This paper described a pilot study for identifying factors that determine the icon feedback usability on small touch screen in four older adult Cognitrone groups since current research aimed mostly at general icon guidelines and recommendations and failed to consider and define the specific needs of small touch screen interfaces for the elderly. In this paper, we presented a concept from the focus on human necessity and use a cognitive assessment tool, which is, Cognitrone test, to measure older adult's attention and concentration capability and learn more about how to evaluate and design suitable small screen icon feedback types. Forty-five elder participants were participated. Each subject was asked to complete a battery of Cognitrone tests and divided into 4 groups. Each subject was also requested to perform a set of 'continuous touch' usability tasks on small touch screen and comment on open-ended questions. Results are discussed with respect to the perceptual and cognitive factors that influence older adults in the use of icon feedback on small touch screen. It showed significant associations between icon feedback performance and factors of attention and concentration. However, this interrelation was much stronger for the Group 2 and Group 4, especially for Type B, Type C and Type G. Moreover, consistent with previous research, older participants were less sensitive and required longer time to adapt to the high-detailed icon feedback. These results are discussed in terms of icon feedback design strategies for interface designers.
\end{abstract}

Keywords: small touch screen, icon feedback, older adults, cognitrone style. 


\section{Introduction}

Lately, "touch" becomes one of the buzzwords. In fact, for over a decade, touch screen technology and devices are in widespread use, from public systems such as self order and information kiosks to personal handheld devices like PDAs (Personal Digital assistants) or gaming devices. Generally speaking, interactions on touch sensitive screens is one of the most "direct" application forms of HCI (Human Computer Interaction), with information and control displayed on one surface. So to say, the zero displacement between input and output, control and feedback, hand movement and eye gaze makes touch screen an intuition-oriented tool for users, particularly for the novices [6]. Nonetheless, as this touch technology gains sophistication and its teething problems being worked out, small touch screen technology meets two limitations. First, the screen might be obscured by the user's finger, hand, or arm. Second, it is difficult for users to point at targets within their finger width. Recently, some studies on thumb use recommended that $9.2 \mathrm{~mm}$ is the most proper width for on-screen icons [2]. Below $9.2 \mathrm{~mm}$, users' performance tends to degrade when they attempt to correctly select an icon on the screen with their thumb. Though the problem can be solved by applying other aids, such as a stylus or a cursor, the easy-to-operate characteristic of thumb-based screen touch no longer exists. Moreover, a practical designer may consider icons of $9.2 \mathrm{~mm}$ too large and space occupying. Therefore, techniques like Offset Cursor and Shift are introduced to improve selection accuracy and to help users to refine their initial selection position. Originally designed for fingertip operation, Offset Cursor overcame digit occlusion by offsetting the cursor from the selection point while Shift achieved it by displaying an inset of selection region.

However, both novel designs are of little significance in their adaptabilities to the altering needs of the elder users as their abilities decline because of aging. Known as the most frequently applied approach for human computer interface design, Nielsen's outlines of the User-centered Paradigm (1993) were intended for homogeneous groups to test the users regarding to design decisions. Yet, the current interface development tools and methods neither meet the needs of diverse user groups, nor do they address the dynamic nature of diversity. As a result, there is an urgent need to implicit the issues of these shortcomings of the current approach as well as to search for new processes and practices.

By its literal definition, touch screen operation is different from normal screen operation. Besides visual search, "touch" actions are involved during the interaction as well. That is to say, the main objective of interface designers is to create a highly user-friendly interface while confirming appropriate design concepts. With the view that older adults' attention ability could be a strong impact factor to their feedback perception of the icon, this study aimed to investigate how icon feedback types affect diverse elderly users when they operate on small touch screen. By generalizing the older generation's perceptions and performances of varied icon feedbacks on small touch screen, analyzing their preferences of different icon feedbacks and finally generating performances and differentiating advantages, the findings of this study served as a guide to icon feedback design of more user- friendly small touch screen. 


\section{Literature Review}

Research on the use of alternative feedback modalities focused primarily on the use of single feedback, while comparatively few studies examined and compared different visual icon feedback combination on small touch screens. As Leonard et al. [4] pointed out in 2006 that additional research is needed to examine specific combined icon feedbacks and their usability for the elderly with varied physical and psychological conditions.

Although passive touch screens are intuition-oriented and easy-to-learn, there are several limitations about the precision that users have to overcome in the interaction. First of all, for touch screens, finger pointing selection of rather small objects and specification of smaller targets may be difficult and critical in effective selections. Second, for interaction on small touch screens, complications may occur, due to occlusion, imprecision in selection, poor calibration, or parallax errors caused by the offset between the display surface and the overlaid touch surface. Third, for touch screen interaction, which is different from the use of mouse, no analogue is involved. Unlike mouse users who can move the mouse pointer over screen elements, get feedback from the selected elements such highlighting as well as confirm their selection by clicking mouse buttons, touch screen users point on screen elements directly and immediately initiate an action which might not be able to canceled afterwards. Fourth, touch screen interaction is characterized by the user's habits and characteristics. In other words, it is a procedure requiring crucial cognitive skills such as concentration, coordinated reactions, excellent judgment together with decision-making capabilities to avoid mistaken manipulation.

Finally, owing to the fact of physical and intellectual declinations of human aging, older adults face more difficulties in small touch screen operation which is intended majorly for the younger users. For instance, when operating with complicated interfaces, it may be hard for the elderly to press minute buttons and detect icon feedbacks because of their varied attention capabilities and habits formulated [3]. Hence, indications whether an action is possible or not have to come along with the icon feedbacks. Likewise, static activation takes more of the elder users' attention for clarifications and action-receiving checks. To sum up, despite the fact that relatively few attempts were made, discussions and studies of icon feedback types are of essential importance. Meanwhile, for small touch screen assessments, direct applications of icon feedback are required, especially those in terms of the estimated numbers of potentially-excluded population and potentially-challenged population among various target population groups.

\section{Methodology}

\subsection{Participants}

Forty-five volunteers ranging from the age of fifty-five to seventy-three years old participated in this study. Among them, twenty-eight were female and seventeen were 
male. The mean age is 67.6 years old. Compensation for participants in this study included free comprehensive cognitrone tests and souvenir. Participants were randomly selected in Taichung City.

\subsection{Cognitrone Test}

The Cognitrone (COG) Test is a general performance test regarding to attention and concentration measurement and analysis, which consists two basic concepts. First, its stimulus materials are composed of stick figures and require paticipants' judgment to decide identical pictures. Second, the Cognitrone test is used for measuring executive functionings such as decision or judgment-making on a person's receptive response to minute changes. Moreover, the COG test is applied in predicting concentration levels and attention spans, which are essential to underground work skills. With a set time limit, participants are asked to accomplish tasks which are not intellectuallydemanding, with possible speed and accuracy.
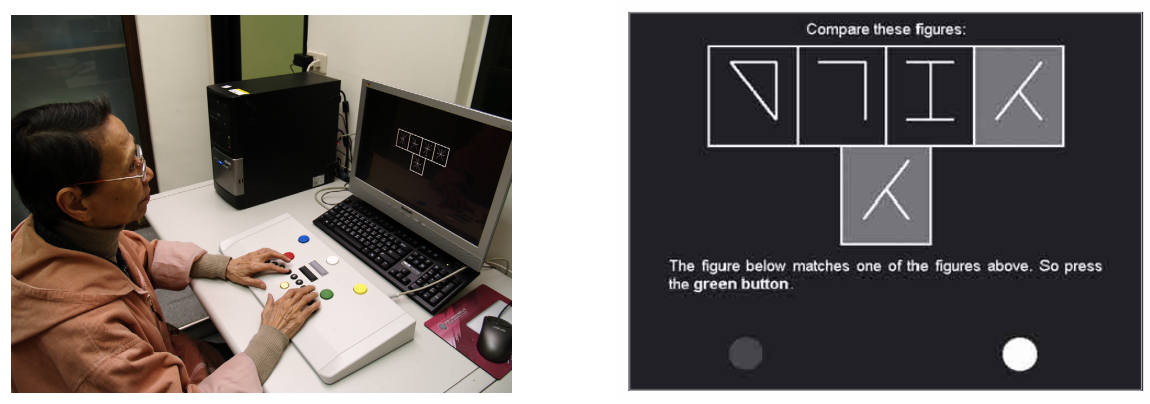

Fig. 1. The Cognitrone test introduction

During the test, participants have to compare the cognitrone of figures. Altogether five pictures are presented on the screen in which four pictures in one line with the fifth picture below them. Participants have to determine whether it is an exact match or not by pressing two different colors of buttons on the response panel. Green buttons are for exact matches while red buttons are for inexact matches. Then, complied measurements describing the subject's performance of speed, accuracy and consistence will be processed and calculated by a scoring program. In regards with the time limit, usually the COG test gives an unlimited completion time. However, it is suggested that the ideal time for completion is between five to ten minutes. Any time longer than ten minutes is considered a reflective of a concentration deficit. Furthermore, the reliability of the COG test is considerably high, which is above $r=9.5$, with a number of validity tests carried out. Meantime, although different versions of COG test are available, the S11 Version is the most suitable for the use of Taiwanese participants because the S11 Version was developed with relevant and applicable norms nation-wide. In this session, participants were divided into four COG groups, each with different analysis result and different focuses on a major trait of attention, as illustrated in Table 1 below. 
Table 1. Arrangements: participants group the number of the participants

\begin{tabular}{c|c|c|c|c}
\hline item & age & gender & \multicolumn{2}{|c}{ Group characteristic } \\
\hline Group1(n=11) & $62.0(3.4)$ & $\mathrm{M}=2 \mathrm{~F}=9$ & Accurate-fast & Accurate-slow \\
\hline Group2(n=15) & $67.3(5.4)$ & $\mathrm{M}=10 \mathrm{~F}=5$ & Inaccurate-fast & \\
\hline Group3(n=12) & $67.2(5.1)$ & $\mathrm{M}=5 \mathrm{~F}=7$ & Inaccurate-slow & inaccurate \\
\hline Group4(n=7) & $66.8(5.5)$ & $\mathrm{M}=0 \mathrm{~F}=7$ & fow \\
\hline
\end{tabular}

\subsection{Materials and Experimental Design}

The interface platform used in this study was an ASUS MyPal A730W compatible PDA. Participants were seated approximately thirty centimeters from the screen display. Simulated screen resolution was set at 1024 x 768 pixels, with a 24-bit color setting. To accomplish continuous-touch tasks, participants were requested to perform a series of random input of ten-digit telephone number. In the meantime, by using the

Table 2. icon feedback example screen shot and experiment scene

\begin{tabular}{l|l|l}
\hline type & \multicolumn{1}{|c}{ Feedback Form Description } \\
\hline $\mathrm{A}$ & $\begin{array}{l}\text { Movement: The position of the icon will } \\
\text { gradually move after icon is touched } \\
\text { Color :The color of the icon will change } \\
\text { after icon is touched } \\
\text { B }\end{array}$ & $\begin{array}{l}\text { Magnify: The shape of the icon will } \\
\text { change after icon is touched }\end{array}$ \\
$\mathrm{D}$ & $\begin{array}{l}\text { Movement+Magnify: The combined } \\
\text { feedback of the icon will apply after icon } \\
\text { is touched }\end{array}$ \\
$\mathrm{E}$ & $\begin{array}{l}\text { Movement+Color: The combined } \\
\text { feedback of the icon will apply after icon } \\
\text { is touched }\end{array}$ \\
$\begin{array}{l}\text { Color+ Magnify: The combined } \\
\text { feedback of the icon will apply after icon } \\
\text { is touched }\end{array}$ \\
$\begin{array}{l}\text { Movement+ Color+ Magnify The } \\
\text { combined feedback of the icon will } \\
\text { apply after icon is touched }\end{array}$
\end{tabular}


Flash X programming language, a group of icon feedback presentations was developed in this study. Also, based on the findings of related research, an average icon size of 6 $\mathrm{mm}$ was adopted in this experiment. Finally, this study employed a 7 x 4 factorial design with seven feedback modality conditions among the four groups of participants illustrated in Table 1 . In addition, two measurements of efficiency were used to assess participants' performance. One was the total time for completion, which was measured in seconds and the other was the frequency of errors of missing or wrong. Both measurements focused on interrelated components of the continuous touch task, which were influenced mostly by the user's response to the icon feedback on small touch screen.

\subsection{Procedures}

Before the experiment, the participants were briefed about the rules and the purpose of the experiment and were requested to fill in their personal information such as their age, gender, and education. In the test session, in order to get accustomed to the interface, the participants were asked to make a simple trial before the start of each task. As the task began, the participants were asked to touch the icon from the program instruction, which adopted progressive interaction in the experiment interface. After that, to complete, the participants had to touch every icon on the touch screen and complete the ten-digit telephone number trials as shown in Table 2. During the trials, the participants experienced all of the seven icon feedback types, which are Type A, Type B, Type C, Type D, Type E, Type F, and Type G. Also, respectively they perceived various usabilities from each icon feedback. At last, after the screen touch tasks being completed, the participants were encouraged to comment on some open-ended questions if there were any aspects for adjustment, improvement or further explanation or if there were any favored features or disliked features. All materials were presented to the participants in Chinese and for the purpose of this paper, all items and questions were translated into English.

\subsection{Data Analysis}

For the analysis of the data, this study applied Analyses of Variance (ANOVA) to examine significant differences of the task performance of feedback conditions within each cognitrone group. When each cognitrone group operated the icon feedback of Type A, Type B, Type C, Type D, Type E, Type F and Type G, the one-way ANOVA analyzed the data. In addition, the significant differences were analyzed by utilizing the Scheffe Method as the post hoc test for multiple comparisons. Significance was accepted at the level of $\mathrm{p}<.05$, while the degrees of freedom and corresponding probability, or the F-value, were also shown in the statistical test. In all, the statistical analysis was conducted by utilizing the Windows SPSS Statistics 17 Program.

\section{Results and Discussions}

The results indicated that changes in overall completion time and the frequency of errors were of highly significance among the four subject groups $(F(3,311)=109.7$, 
$\mathrm{p}<.01$ ). Hence, following the ANOVA analysis of the four groups, Table 3 depicted the mean total time for completion and error frequency of each icon feedback condition. In general, the results of the ANOVA analysis and Figure2, Figure 3, Figure 4 and Figure 5 indicated that different feedback condition had a significant effect on Group 2 and Group 4, as indicated by the test statistics and post hoc test results described in Table 3 below. As to Group 1 and Group 3, no significant effect of feedback condition was appreciated $(\mathrm{p}<.05)$.

To begin with, for Group 1, neither the icon feedback of completed time nor the effect of error frequency was significant, which showed that the older adults in Group 1 had fewer positive errors during touch screen interaction. Regarding their characteristics, they attended to touch icons in a quick review and made a correct decision. For this reason, Group 1 participants were considerably effortless to experience and detect the small-size icon feedbacks. Nonetheless, when compared to the theory of Capability Demand, the demand levels of these icon feedbacks were multidimensional and set by the attributes of small touch screen interface features. Therefore, even if the Group 1 participants had higher performance, other potential factors in terms of creative interaction design methods were still needed further clarification.

Secondly, for Group 2, the results indicated that both total completed time and error frequency were significant among the feedback types. Among them, the icon feedback of Type A, Type D, Type E and Type $G$ were more appreciated as they were two-dimensional effects which provide spatial and semantic cues for the participants. Moreover, as other normal feedback application intensified in Type B and Type C, the participants' response with a slower rate for task completion, which was an indication that those individuals with G2 characteristics do not benefit from the change of color or shape. In other words, the findings suggested that for small touch screen, concentration on location changes are highly required since Group 2 participants preferred spatial and semantic cues to organize information. Hence, designs of icon feedback should take their specific requirements into consideration.

Although for Group 3 there was not much significance appreciated on the icon feedback types, still the findings were useful. It is agreed that an ideal icon feedback design not only pay more attention to older adults with declining attention capability but also add more chance for the elderly to improve their performance. Take Group 3 participants for example, they perceived icon feedbacks in a rather short time and made more mistakes. For them, the icon feedback design should shift its focus to the concept of shape-change because structural components were regarded as a perceiving modal of information transferring. Thus, the conceptualization of improved icon feedback with alerting function may be an insightful solution to this issue concerned.

Still, in regards to Group 4, the findings showed that participants prefer external structure presented by the material in the Type D and Type $G$ interactions, which was consistent with the findings of the previous studies in the domains of user capabilities. [5] That is, for some older adults, more interaction steps and thinking time for small screen interaction were needed, especially for diverse characteristics of the older 
Table 3. Results of operational time and frequency of errors on icon feedback type

\begin{tabular}{|c|c|c|c|c|c|c|}
\hline Group & & $\mathrm{df}$ & Mean Square & $\mathrm{F}$ & Sig. & Post hoc Tests \\
\hline \multicolumn{7}{|c|}{ Group1 (Accurate-fast) } \\
\hline \multirow[t]{2}{*}{ time } & Between Groups & 6 & 12.4 & \multirow[t]{2}{*}{1.61} & \multirow[t]{2}{*}{0.156} & \multirow[t]{2}{*}{-} \\
\hline & Within Groups & 70 & 7.7 & & & \\
\hline \multirow[t]{2}{*}{ error } & Between Groups & 6 & 0.53 & \multirow[t]{2}{*}{0.97} & \multirow[t]{2}{*}{0.452} & \multirow[t]{2}{*}{ - } \\
\hline & Within Groups & 70 & 0.55 & & & \\
\hline \multicolumn{7}{|c|}{ Group2 (Accurate-slow) } \\
\hline \multirow[t]{2}{*}{ time } & Between Groups & 6 & 68.1 & \multirow[t]{2}{*}{6.24} & \multirow[t]{2}{*}{0.00} & $\mathrm{~A}<\mathrm{C}$ \\
\hline & Within Groups & 98 & 10.9 & & & $\mathrm{D}, \mathrm{E}, \mathrm{F}, \mathrm{G}<\mathrm{C}$ \\
\hline \multirow[t]{2}{*}{ error } & Between Groups & 6 & 12.6 & \multirow[t]{2}{*}{12.6} & \multirow[t]{2}{*}{0.00} & $\mathrm{~A}, \mathrm{D}, \mathrm{E}, \mathrm{F}, \mathrm{G}<\mathrm{C}$ \\
\hline & Within Groups & 98 & 1.0 & & & $\mathrm{~A}, \mathrm{E}, \mathrm{G},<\mathrm{B}$ \\
\hline \multicolumn{7}{|c|}{ Group3 (Inaccurate-fast) } \\
\hline \multirow[t]{2}{*}{ time } & Between Groups & 6 & 3.4 & \multirow[t]{2}{*}{0.39} & \multirow[t]{2}{*}{0.88} & \multirow[t]{2}{*}{-} \\
\hline & Within Groups & 77 & 8.8 & & & \\
\hline \multirow[t]{2}{*}{ error } & Between Groups & 6 & 3.9 & \multirow[t]{2}{*}{2.78} & \multirow[t]{2}{*}{0.01} & \multirow[t]{2}{*}{-} \\
\hline & Within Groups & 77 & 1.4 & & & \\
\hline \multicolumn{7}{|c|}{ Group4 (Inaccurate-slow) } \\
\hline \multirow[t]{2}{*}{ time } & Between Groups & 6 & 26.8 & \multirow[t]{2}{*}{2.64} & \multirow[t]{2}{*}{0.02} & \multirow[t]{2}{*}{$\mathrm{G}<\mathrm{C}, \mathrm{B}$} \\
\hline & Within Groups & 42 & 10.1 & & & \\
\hline \multirow[t]{2}{*}{ error } & Between Groups & 6 & 6.22 & \multirow[t]{2}{*}{5.90} & \multirow[t]{2}{*}{0.00} & \multirow[t]{2}{*}{$\mathrm{G},<\mathrm{B} ; \mathrm{G},<\mathrm{E}$} \\
\hline & Within Groups & 42 & 1.05 & & & \\
\hline
\end{tabular}

population. Altogether, results of this study and the previous works suggested that the elderly tend to require more interaction steps than the other age groups. Moreover, this study also showed that Type D and Type G helped Group 4 participants with available information source by getting an overview of the feedback content via clear movement and magnification method.

Finally, for the open-ended questions about problems and further improvements for the 7 icon feedback types, problems commonly reported by the participants were "I felt a little fatigue when I tried to focus on some icon for a period of time," and "Occasionally, I touched wrong places near my targets." They also gave comments such as "It was easy to find or understand the icon while being touched (especially for the G Type)." The discussions can be developed into an understanding of how icon feedback features of small touch screen are perceived by older adults with different attention capability styles. Furthermore, comparing the related concept in Microsoft touch screen technique such as offset cursor, shift and wedge [7], some sophisticated adjustments to different people and situations could also be further applied. Even though it could be a tiny issue, it could still be an important role for the interaction on small touch screen. 


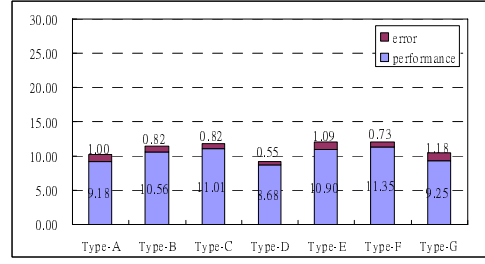

Fig. 2. Group 1 performance means

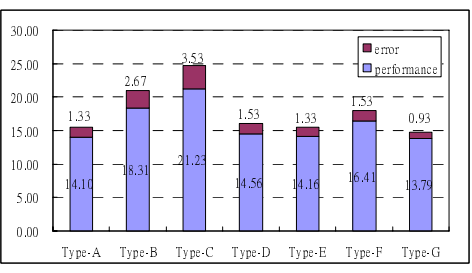

Fig. 3. Group 2 performance means

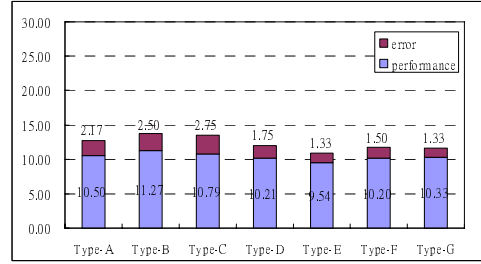

Fig. 4. Group 3 performance means

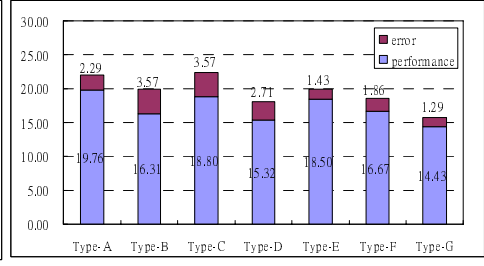

Fig. 5. Group 4 performance means

\section{Conclusion}

This study examined the usability of icon feedback types in small touch screen devices. In order to find out how these feedbacks are suited for different Cognitrone groups, four divided older adult groups were examined. They represented the preferences of each icon feedback type, also they provided an overall picture of users' need while using small touch screen. The findings can be used to develop guidelines for the design of icon feedback that suits the preferences of diverse older adults. More specific design considerations should be taken into consideration when developing a new icon feedback. Further research could also address access to appropriate software technique for touch screen interaction which can be characterized by the other factors, such as icon feedback characteristics on speed, intensity, portion, locomotion, and precision. We hope to provide a capability-diverse framework providing a useful starting point for analytical evaluation, by focusing on ways of related small touch screen issues to the range of user capabilities.

Acknowledgements. This study received partly financial support from the National Science Council of the Republic of China Government, under Grant No. NSC 97-2221-E-224-024.

\section{References}

1. Dickinson, A., Gregor, P.: Computer use has no demonstrated impact on the well-being of older adults. International Journal of Human-Computer Studies 64(8), 744-753 (2006)

2. Jin, Z.X., Kiff, T.P.L.: Touch screen user interfaces for older adults: Button size and spacing. In: The 4th International Conference on Universal Access in Human-Computer Interaction, UAHCI 2007, Held as Part of HCi International 2007, Beijing, China (2007) 
3. Lee, C.F., Tsai, W.C.: Mapping of user interfaces on electronic appliances. Applied Ergonomics 38(5), 667-674 (2007)

4. Leonard, V.K., Jacko, J.A., Pizzimenti, J.J.: An investigation of handheld device use by older adults with age-related macular degeneration. Behaviour \& Information Technology 25(4), 313-332 (2006)

5. Persad, U., Langdon, P., Clarkson, J.: Characterising user capabilities to support inclusive design evaluation. Universal Access in the Information Society 6(2), 119-135 (2007)

6. Shneiderman, B.: Touch screens now offer compelling uses. IEEE Software 8(2), 93-94 (1991)

7. Vogel, D., Baudisch, P.: Shift: a technique for operating pen-based interfaces using touch. In: The 2007 Conference on Human Factors in Computing Systems, San Jose, California, USA (2007) 